\title{
Synchronization of Discrete Oscillators on Ring Lattices and Small-World Networks
}

\author{
Kevin Liu Rodrigues ${ }^{1}$ and Ronald Dickman ${ }^{1}$ \\ ${ }^{1}$ Departamento de Física, Instituto de Ciências Exatas, \\ and National Institute of Science and Technology for Complex Systems, \\ Universidade Federal de Minas Gerais \\ C.P. 702, 30123-970, Belo Horizonte, MG, Brazil
}

(Dated: July 8, 2021)

\begin{abstract}
A lattice of three-state stochastic phase-coupled oscillators introduced by Wood et al. [Phys. Rev. Lett. 96, 145701 (2006)] exhibits a phase transition at a critical value of the coupling parameter $a$, leading to stable global oscillations (GO). On a complete graph, upon further increase in $a$, the model exhibits an infinite-period (IP) phase transition, at which collective oscillations cease and discrete rotational $\left(C_{3}\right)$ symmetry is broken [Assis et al., J. Stat. Mech. (2011) P09023]. These authors showed that the IP phase does not exist on finite-dimensional lattices. In the case of large negative values of the coupling, Escaff et al. [Phys. Rev. E 90, 052111 (2014)] discovered the stability of travelling-wave states with no global synchronization but with local order. Here, we verify the IP phase in systems with long-range coupling but of lower connectivity than a complete graph and show that even for large positive coupling, the system sometimes fails to reach global order. The ensuing travelling-wave state appears to be a metastable configuration whose birth and decay (into the previously described phases) are associated with the initial conditions and fluctuations.
\end{abstract}

PACS numbers: 64.60.Ht, 05.45.Xt, 89.75.-k 


\section{INTRODUCTION}

Systems of coupled oscillators exhibit diverse symmetry-breaking transitions to a globally synchronized state. In the paradigmatic Kuramoto model, for instance, oscillators with distinct intrinsic frequencies $\omega_{i}$ coupled via their continuous phases $\theta_{i}$ can exhibit stable collective oscillations, breaking time-translation invariance [1 15]. Amongst discrete-phase models, the paper-scissors-stone game is an example of a system with three absorbing states that can exhibit either global oscillations or spontaneous breaking of discrete rotational $\left(C_{3}\right)$ symmetry [6 10]. More recently, Wood and coworkers proposed a family of models of phase-coupled three-state stochastic oscillators that undergo a phase transition to a state exhibiting global oscillations (GO) [11-14] for sufficiently strong coupling. We shall refer to these as Wood's cyclic model (WCM). Although the WCM also has $C_{3}$ symmetry, it has no absorbing state. In addition to their intrinsic interest in the context of nonequilibrium phase transitions, this family of models serve as a highly simplified description of collective neuronal behavior.

The first WCM [11] was found to undergo a second phase transition upon further increase of the coupling [15], at which the period of oscillation becomes infinite, thereby breaking $C_{3}$ symmetry. In [15], this infinite-period (IP) transition was studied on a complete graph (all-to-all coupling), and a novel order parameter, involving the mean rate of change of the probability distribution, was proposed. On the basis of a nucleation scenario, the authors of Ref. [15] argued against the existence of an IP transition on finite-dimensional lattices with short-range interactions, but left open the question of its occurrence on networks with nonlocal interactions. Here we study a WCM on (1) regular rings with interactions up to $2 K$-neighbors, varying the interaction range $K$, and (2) small-world networks. Using numerical simulations to study the order parameter and its variance, we verify the existence of GO and IP phase transitions on these structures. For regular rings of $N$ nodes, the degree of connectivity is characterized by $\alpha \equiv K / N$ such that $\alpha \in(0,0.5)$. A key question is the minimum value of $\alpha$ necessary to observe the GO and IP phases as $N \rightarrow \infty$. Our results suggest that any $\alpha>0$ is sufficient.

We also provide evidence that for intermediate interaction ranges, global synchronization depends sensitively on initial conditions: Some realizations with a random initial configuration show no global synchronization. Such events persist even as the number of neighbors 
grows in proportion to the system size. In this situation, the final state may be a travelling wave, as observed by Escaff et al. [16] in the case of anti-crowding, i.e., interactions favoring anti-synchronization between neighbors.

The remainder of this paper is organized as follows. In section [I] we review the WCM and the essentials of the transitions to the GO and IP phases. We report our results on the GO and IP phase transitions on regular rings, and on small-world networks, in sections III] and IV, respectively. Our conclusions are discussed in section $\mathrm{V}$.

\section{MODEL}

In the WCM, the state $j_{x}$ at site $x(x=1, \ldots, N)$ can take one of three values, $j_{x} \in$ $\{0,1,2\}$, corresponding to a phase $\phi_{x}=2 \pi j_{x} / 3$. The only allowed transitions are those from $j$ to $j+1$ (modulo 3) (see Fig. 1), which implies that detailed balance is violated. If site $x$ is in state $j$, its transition rate to state $j+1$ is:

$$
g_{j, j+1}=g \exp \left[\frac{a\left(n_{x, j+1}-n_{x, j}\right)}{z}\right]
$$

where $g$ is a constant rate, $a$ is the coupling parameter, $n_{x, k}$ is the number of nearest neighbors of site $x$ in state $k$, and $z$ is the number of nearest neighbors. Since these rates are invariant under cyclic permutation of the state indices, the model is invariant under the group $C_{3}$ of discrete rotations.

Let $N_{j}$ be the total number of sites in state $j$, so that $N_{0}+N_{1}+N_{2}=N$, the total number of sites. As discussed in [15], the mean-field (MF) approximation, obtained by replacing $n_{x, j} / z$ in the argument of the exponential of Eq.(1) with the corresponding

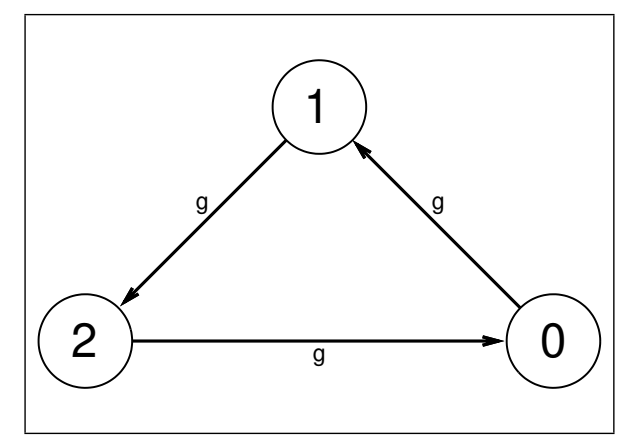

FIG. 1. Transition rates for an isolated unit. 

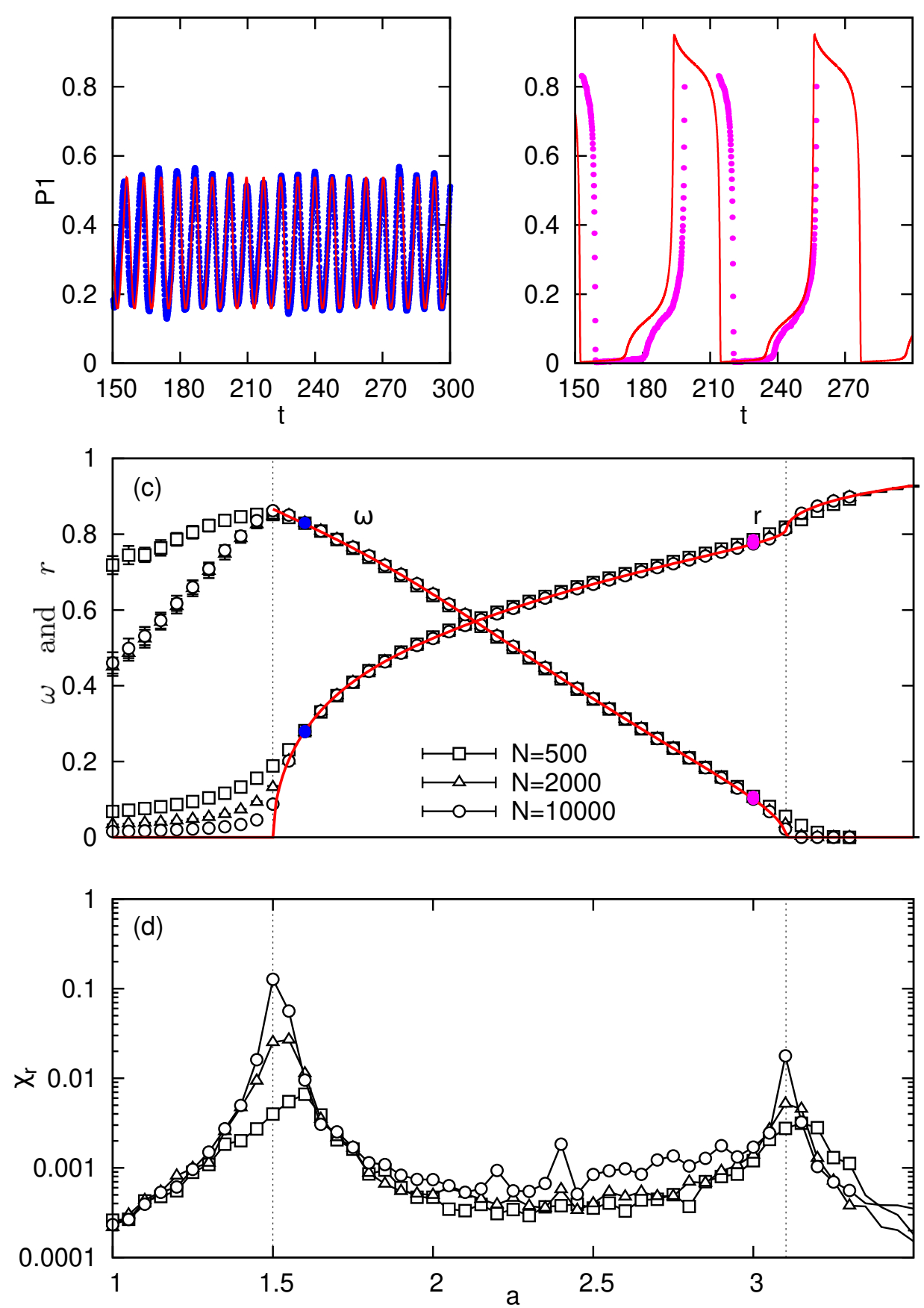

FIG. 2. (Color online) Panels (a) and (b) show the evolution of $P_{1}$ for $a=1.6$ and $a=3$, respectively. Points: simulations of a complete graph of $N$ nodes; lines: mean-field solution. (c) Dependence of $r$ and $\omega$ on $a$, exhibiting the two phase transitions. (d) $\chi_{r}$ versus $a$, showing peaks at the transitions [system sizes as in (c)]. 
state fraction, $n_{j}=N_{j} / N$, yields three nonequilibrium phases, separated by two continuous phase transitions. For small coupling $\left(a<a_{c}=1.5\right)$, the disordered phase, with $\mathbf{n}=(1 / 3,1 / 3,1 / 3) \equiv \mathbf{n}_{1 / 3}$, is the stable stationary solution of the MF equations. (n denotes the vector of state fractions.) For $a$ between $a_{c}$ and a higher value, $a^{c} \simeq 3.10243991564$, there is no stable stationary solution and the MF equations admit an oscillatory solution (a limit cycle) in which states 0,1 and 2 periodically assume the role of the majority. As $a$ is increased above $a_{c}$, the frequency $\omega$ of oscillation decreases continuously, becoming zero at $a^{c}$, signalling the IP transition. For $a>a^{c}$, three stationary solutions, $\mathbf{n}_{i}$ appear, such that state $i$ represents the (permanent) majority. Thus $C_{3}$ symmetry is broken for $a>a^{c}$. (The three solutions are, naturally, related via cyclic permutation of indices in state space.)

The WCM is characterized by a pair of order parameters. First, one has the familiar Kuramoto synchronization parameter [1, 3, 11],

$$
r \equiv\left\langle\langle|v|\rangle_{t}\right\rangle_{s},
$$

where

$$
v \equiv \frac{1}{N} \sum_{x=1}^{N} e^{i \phi_{x}} .
$$

In Eq. (2), \langle\rangle$_{t}$ denotes a time average over a single realization (in the stationary state), and \langle\rangle$_{s}$ an average over independent realizations. Note that $r>0$ is consistent with, but does not necessarily imply, globally synchronized oscillation. The latter is characterized by a periodically varying phase of $v[17-20]$.

In the MF analysis, the transition to the synchronized regime (the GO transition) is associated with a supercritical Hopf bifurcation at $a=a_{c}=1.5$ : the trivial fixed point $\mathbf{n}_{1 / 3}$ loses stability at $a=a_{c}$, and a limit cycle encircling this point appears. For $a \gtrsim a_{c}$, sustained oscillations in $n_{j}$ characterize synchronization among the oscillators (Fig. 2a). Correspondingly, $r$ grows continuously $\sim\left(a-a_{c}\right)^{\beta}$ at the transition (Fig. 2 2 ), with a meanfield exponent $\beta=1 / 2$ [11]. The scaled variance

$$
\chi_{r} \equiv L^{d}\left[\left\langle\langle|v|\rangle_{t}^{2}\right\rangle_{s}-r^{2}\right],
$$

diverges with the system size at criticality, as shown in Fig. 20 for simulations on the complete graph [15]. The GO transition is associated with breaking of the continuous timetranslation symmetry: the $n_{j}(t)$, are periodic for $a \gtrsim a_{c}$. Increasing $a$ above $a_{c}$ enhances 
synchronization among the oscillators, leading to increasing oscillation amplitudes, as shown in Fig. 2b.

Wood et al. found that the increasing amplitude of oscillation is accompanied by a decreasing angular frequency $\omega=2 \pi /\langle\tau\rangle$, where $\langle\tau\rangle$ is the mean time between peaks in $n_{k}$ (Figs. 2a-c). This can be understood qualitatively from the exponential dependence of the transition rates of Eq. (1) on the neighbor fractions: When a state is highly populated, the rate at which oscillators leave it becomes very small. In mean-field theory, when $a$ reaches the upper critical value $a^{c}$, three symmetric saddle-node bifurcations occur simultaneously, and the period of the collective oscillations diverges [15]. Above $a^{c}$, there are three symmetric attractors in the system, and 3-fold rotational $\left(C_{3}\right)$ symmetry is spontaneously broken. As in condensation or a ferromagnetic phase transition [21], freezing of the majority state does not imply that individual oscillators freeze as well. The transition rates of individual oscillators do decrease with increasing $a$, but only vanish in the limit $a \rightarrow \infty$, when one of the states is fully occupied.

It is convenient to define an order parameter $\psi$ that is identically zero (in the infinite-size limit) for $a>a^{c}$. Assis et al. proposed [15],

$$
|\psi| \equiv \frac{1}{N}\left|\sum_{x=1}^{N}\left(\delta_{0, j_{x}}+e^{2 \pi i / 3} \delta_{1, j_{x}}+e^{-2 \pi i / 3} \delta_{2, j_{x}}\right) \gamma_{x}\right|
$$

where $\delta_{i j}$ is the Kronecker delta and $\gamma_{x} \equiv g_{j_{x}, j_{x}+1}$ is the transition rate at site $x$ (see eq. 1). Thus $|\psi|$ involves not only the configuration, but the rate at which the latter evolves. On the complete graph, $\gamma_{x}$ is the same for all sites $x$ in the same state $j$. Denoting this rate by $\gamma_{j}$, the order parameter can be written (in MF analysis) as

$$
|\psi|^{2} \stackrel{M F}{=} \sum_{j=0}^{2}\left(n_{j} \gamma_{j}-n_{j-1} \gamma_{j-1}\right)^{2}
$$

Both the disordered phase $\left(a<a_{c}\right)$ as well as the IP phase $\left(a>a^{c}\right)$ have stable, stationary solutions, $\mathbf{n}^{*}$. Since $\dot{n}_{j}=0$ implies $n_{j}^{*} \gamma_{j}=n_{j-1}^{*} \gamma_{j-1}$, i.e., zero net change in the probability of state $j$, we have $|\psi|=0$ in eq. 6 for both cases [a similar line of reasoning can be applied directly to Eq. (5)].

Figure 3 shows $\langle|\psi|\rangle$ versus $a$ in MF theory, and on the complete graph (the latter via 


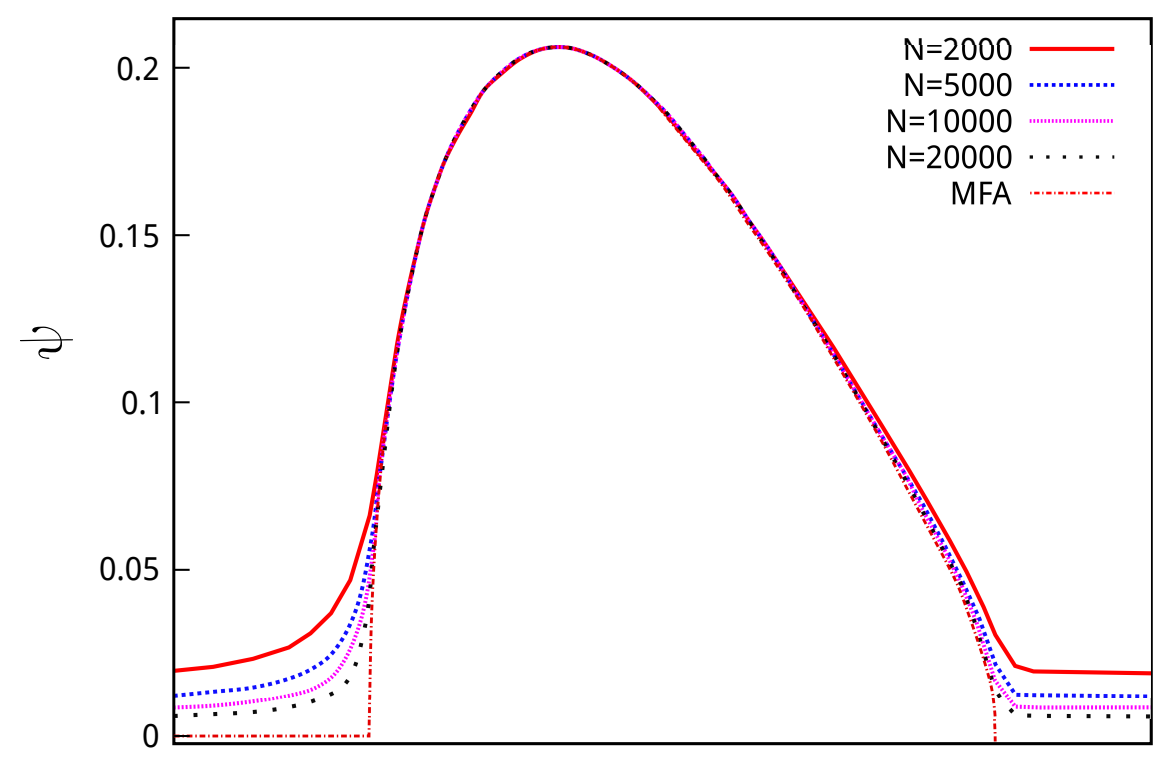

a

FIG. 3. (Color online) (From [15].) Order parameter $\langle|\psi|\rangle$ as a function of coupling $a$ in mean-field theory and on the complete graph, for sizes as indicated.

numerically exact solution of the master equation), confirming that $|\psi|$ functions as an order parameter to detect both the GO and IP phase transitions. The MF critical behavior is $\langle|\psi|\rangle \sim\left(a-a_{c}\right)^{1 / 2}$ for $a \searrow a_{c}$ and $\langle|\psi|\rangle \sim\left(a^{c}-a\right)^{1 / 2}$ for $a \nearrow a^{c}$. On the complete graph, the order parameter decays with system size as $\langle|\psi|\rangle \sim N^{-1 / 4}$ at $a=a_{c}$ and as $N^{-0.4203(3)}$ at $a=a^{c}$. The first result is typical of mean-field-like scaling with system size at a continuous phase transition, as argued in [15].

The results for the IP transition in MF and on a complete graph are in sharp contrast to what is found on finite-dimensional lattices. The absence of such a transition was verified numerically on hypercubic lattices in dimensions $d \leq 4$ in [15]. This reference also provides a quantitative argument showing that on finite-dimensional lattices, a $j$-state majority cannot persist indefinitely: it is always susceptible to change via nucleation of a cluster of state $j+1$. The authors of [15] conjectured that the IP transition would occur on structures in which a site interacts with a nonzero fraction of all other sites (as the system size tends to infinity). In the following sections we test this conjecture on two structures, regular rings with extended interactions, and small-world networks. 


\section{THE WCM ON REGULAR RINGS}

A regular ring is constructed starting from a graph of $N$ nodes arranged in circular fashion. Considering one node at a time in a clockwise manner, we connect it to its $K$ nearest neighbors in the clockwise direction; an example of such a structure is shown in Fig. 4. We define the connectivity of a regular ring graph by $\alpha \equiv K / N$, such that $\alpha=1 / N$ signifies a one-dimensional chain while $\alpha=0.5$ represents a complete graph. Thus, one can interpolate from the one-dimensional to an infinite-dimensional hypercubic lattice (complete graph) varying $\alpha$ over the interval $\left(0, \frac{1}{2}\right]$. It is known that the WCM on hypercubic lattices of dimensions 1 and 2 cannot sustain ordered phases [11, 15]. Since $\alpha=0.5$ represents the complete graph, there must be at least one threshold $\alpha=\alpha^{*}$ above which one or both phase transitions (GO and IP) occur, varying $a$.

Different from hypercubic lattices, in which coupling is local, for $\alpha>0$ the coupling on ring graphs is nonlocal. The manner in which the interaction range scales as $N \rightarrow \infty$ can be chosen in different ways; the simplest, which we consider here, is to fix $\alpha$ so that $K \propto N$ (More precisely, $K=\lceil\alpha N\rceil$ where $\lceil x\rceil$ denotes the smallest integer larger than $x$ ). It is reasonable to expect that phase transitions occur for any fixed $\alpha>0$, since the interaction range $K$ then tends to infinity with $N$.

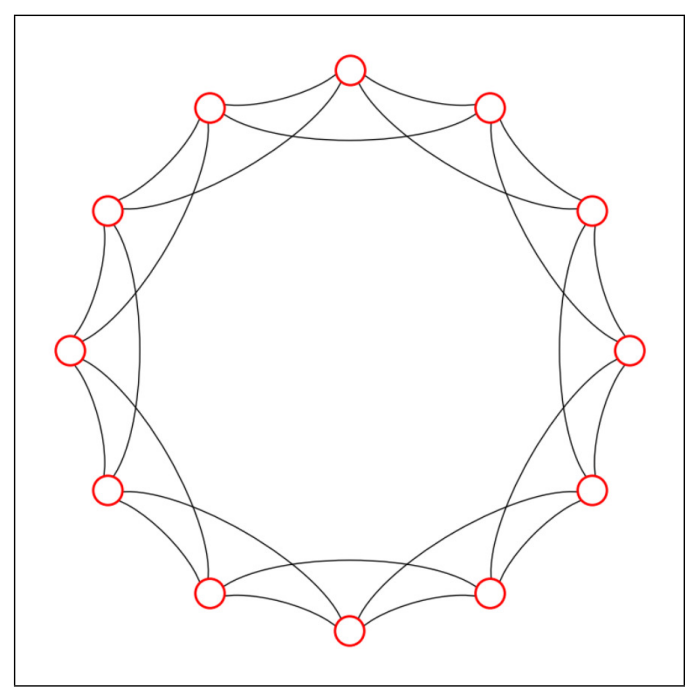

FIG. 4. A regular ring is an undirected graph with $N$ nodes arranged in circular fashion, with each connected to its $K$ nearest neighbors in each direction. Here we show an example with $N=12$ and $K=2$. 

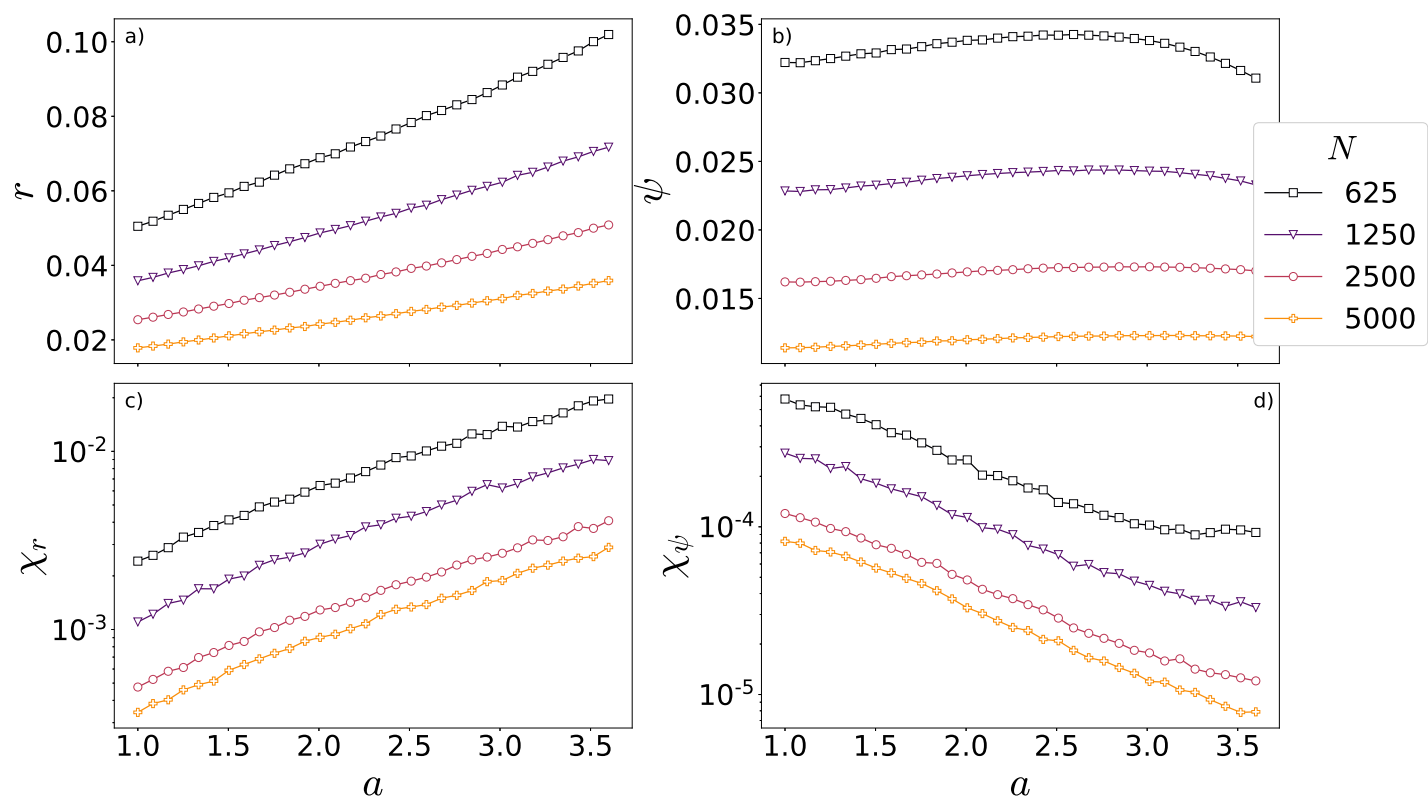

FIG. 5. (Color online) Order parameters and their scaled variances for one-dimensional rings $(K=1)$. Both the order parameters and their respective variances decrease as the system size is increased, with $r \approx \psi \approx 0$ across a wide range of coupling strengths. Points represent an average over 3000 independent realizations with random initial configurations. For the 1D chain, the same behavior is observed regardless of initial configuration.

\section{A. Scaling behavior: phase boundary}

The dynamics as $N$ increases can be studied by defining the scaled variances of the order parameters. These quantities are expected to diverge in the thermodynamic limit when the system undergoes a continuous phase transition [22]. The scaled variances of order parameters $r$ and $\psi$ can be defined through equations 3 and 5 as:

$$
\begin{aligned}
& \chi_{r}=N\left[\left\langle\langle|v|\rangle_{t}^{2}\right\rangle_{s}-\left\langle\langle|v|\rangle_{t}\right\rangle_{s}^{2}\right], \\
& \chi_{\psi}=N\left[\left\langle\langle|\psi|\rangle_{t}^{2}\right\rangle_{s}-\left\langle\langle|\psi|\rangle_{t}\right\rangle_{s}^{2}\right],
\end{aligned}
$$

where \langle\rangle$_{t}$ and \langle\rangle$_{s}$ are averages over time and over independent realizations, respectively.

In simulations, the system is allowed to relax to a steady state, starting from its initial configuration. Once the steady state has been attained, the order parameters are averaged over the remainder of the evolution. As we will see, the choice of initial condition is important 

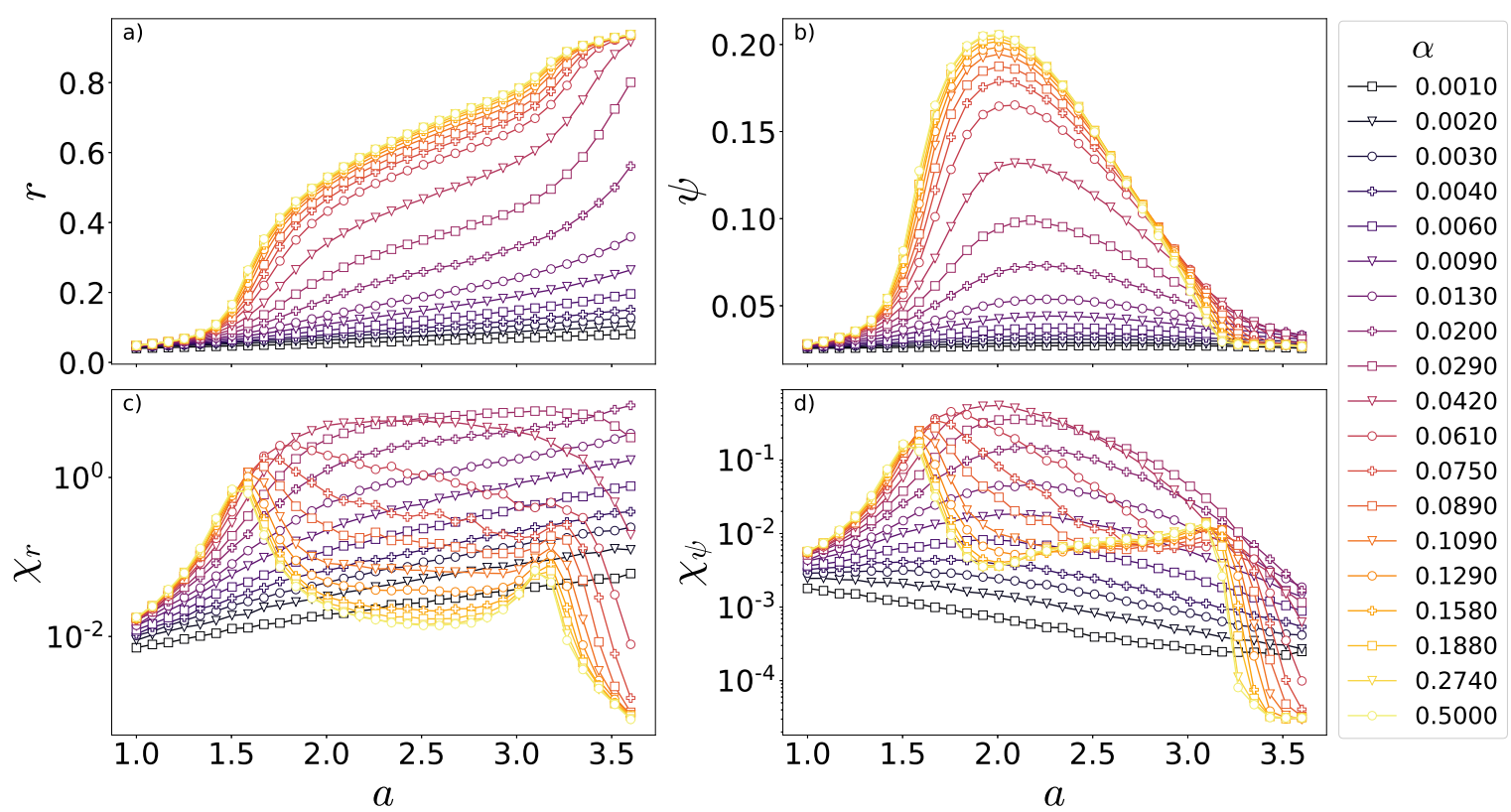

FIG. 6. (Color online) Order parameters and scaled variances for regular rings of size $N=1000$ and various values of the connectivity $\alpha$. Points represent an average over 4000 independent realizations with uniform initial configurations.

for some values of the interaction range $K$; we will focus on two different setups: random initial configurations, in which the initial phase of each oscillator is chosen uniformly and independently among the three possible values in $\{0,2 \pi / 3,4 \pi / 3\}$, and a uniform initial condition, in which $\phi_{i}=0, \forall i$.

In the following we describe results for uniform initial conditions. In Fig. 5 we plot the order parameters and their associated variances for $K=1$ (i.e., $\alpha=1 / N)$ ). Both quantities are shown to decrease with system size (for the 1D case in particular this behavior is the same regardless of initial configuration), indicating the absence of phase transitions. In Fig. 6, the same quantities are shown for regular rings with $N=1000$ and various $\alpha$ values. As expected, the order parameters and their variances approach the complete-graph limit as $\alpha$ nears the value $1 / 2$. Denoting by $\alpha^{*}$ the value associated with a change from one to two maxima in $\chi_{\psi}$, we identify $\alpha^{*} \approx 0.06$ for $N=1000$ in Fig. 6. Performing similar analyses for different system sizes we obtain $\alpha^{*}$ as a function of $N$. To infer the scaling behavior, we define $\lambda \equiv N^{-1}$ and look at the $\alpha^{*}$ versus $\sqrt{\lambda}$ curve near $\lambda=0$. The resulting data, shown in Fig. 7, suggest that $\alpha^{*}$ tends to zero as $N \rightarrow \infty$. This supports the conjecture stated 


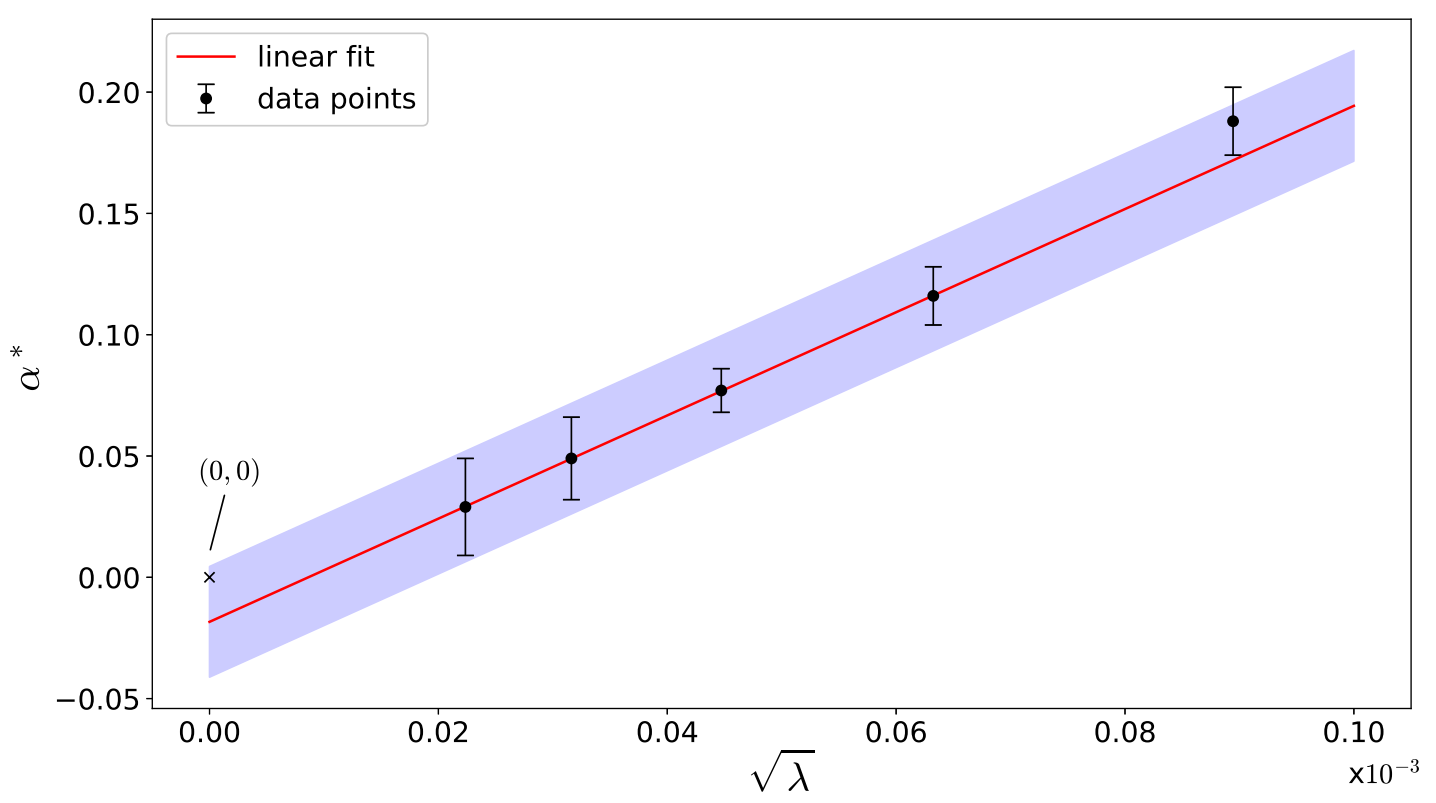

FIG. 7. Plot of $\alpha^{*}$ versus $\sqrt{\lambda}$. Starting from uniform initial configurations, $\alpha>\alpha^{*}$ means $\chi_{\psi}$ and $\chi_{r}$ exhibit two maxima, whereas for $\alpha<\alpha^{*}$ only a single broad maximum is observed. Full circles represent data obtained from simulations and solid curve is a linear fit with equation $y=-0.01837+2.1271 x$. The band around the fitted curve represent the uncertainty associated with the linear intercept.

previously that in this limit, and for any fixed $\alpha>0$, the WCM on a regular ring lattice exhibits both GO and IP phase transitions.

\section{B. Scaling behavior: order parameter}

To better understand the scaling behavior we look at the order parameters as $N \rightarrow \infty$ with fixed $\alpha$. If both $r$ and $\psi$ tend to zero, there is no global synchronization. Both order parameters tending to positive values indicates the presence of global or intermittent synchronization among large populations of oscillators, while $\psi \rightarrow 0$ with $r \sim 1$ defines an infinite-period phase.

In this context it is useful to plot the order parameter versus $\lambda \equiv 1 / N$. An upward (downward) curvature as $\lambda \rightarrow 0$ signals a nonzero (zero) value of the order parameter. In 

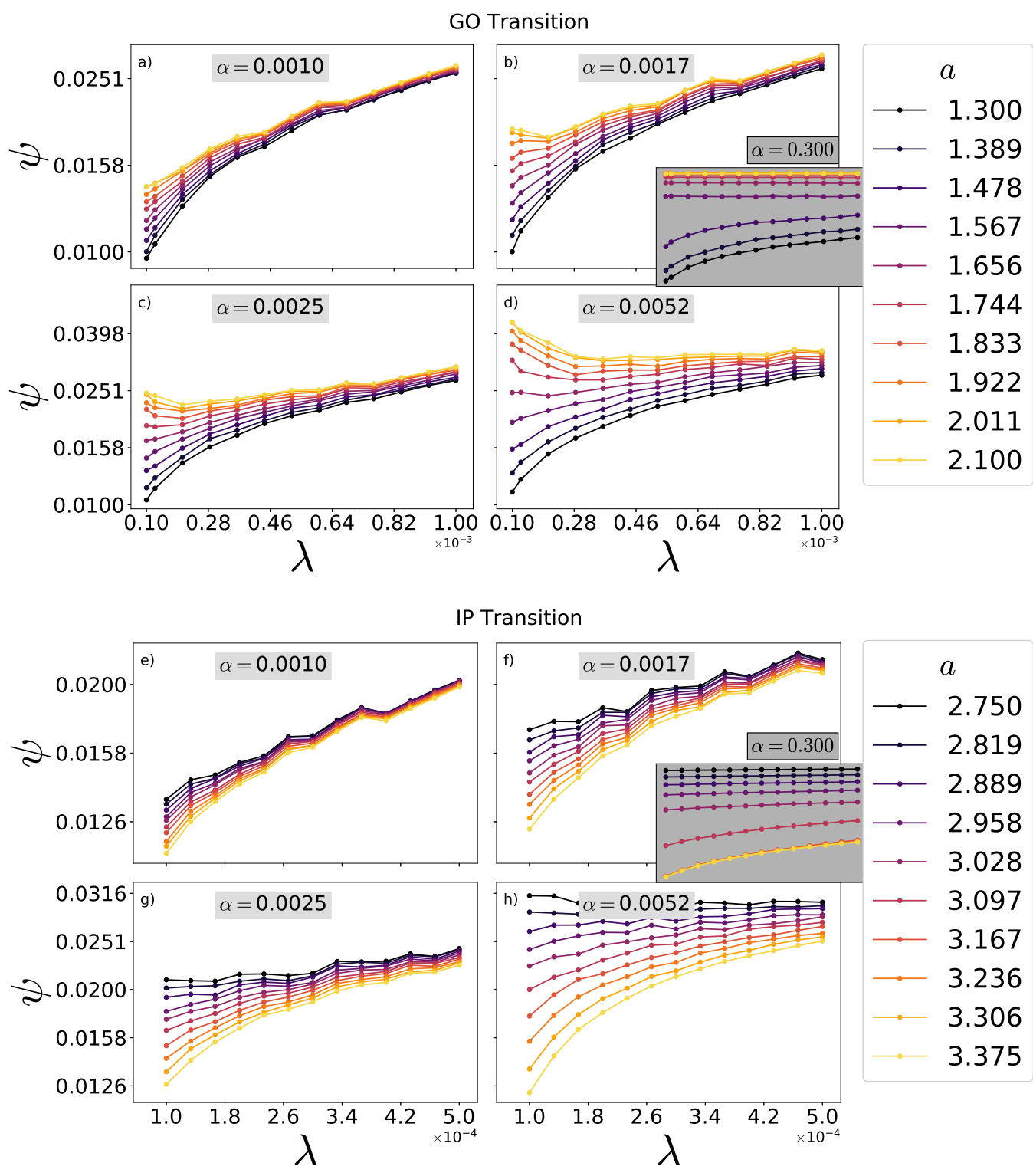

FIG. 8. (Color online) Order parameter $\psi$ versus inverse system size $\lambda$ for various values of $\alpha$, and coupling strengths $a$ in the vicinity of the GO and IP phase transitions. The insets in $\mathbf{b}$ and f show the behavior for $\alpha=0.3$, approaching the complete graph, with GO and IP transitions near $a_{c}=1.5$ and $a^{c} \approx 3.1$ respectively. Each point represents an average over 400 independent realizations. The oscillatory behavior seen in some panels is due to the reduction in average path lengths caused by the introduction of new neighbors as the system size increases at fixed $\alpha$, and the fact that $K$ must be an integer (see Appendix VIA). 

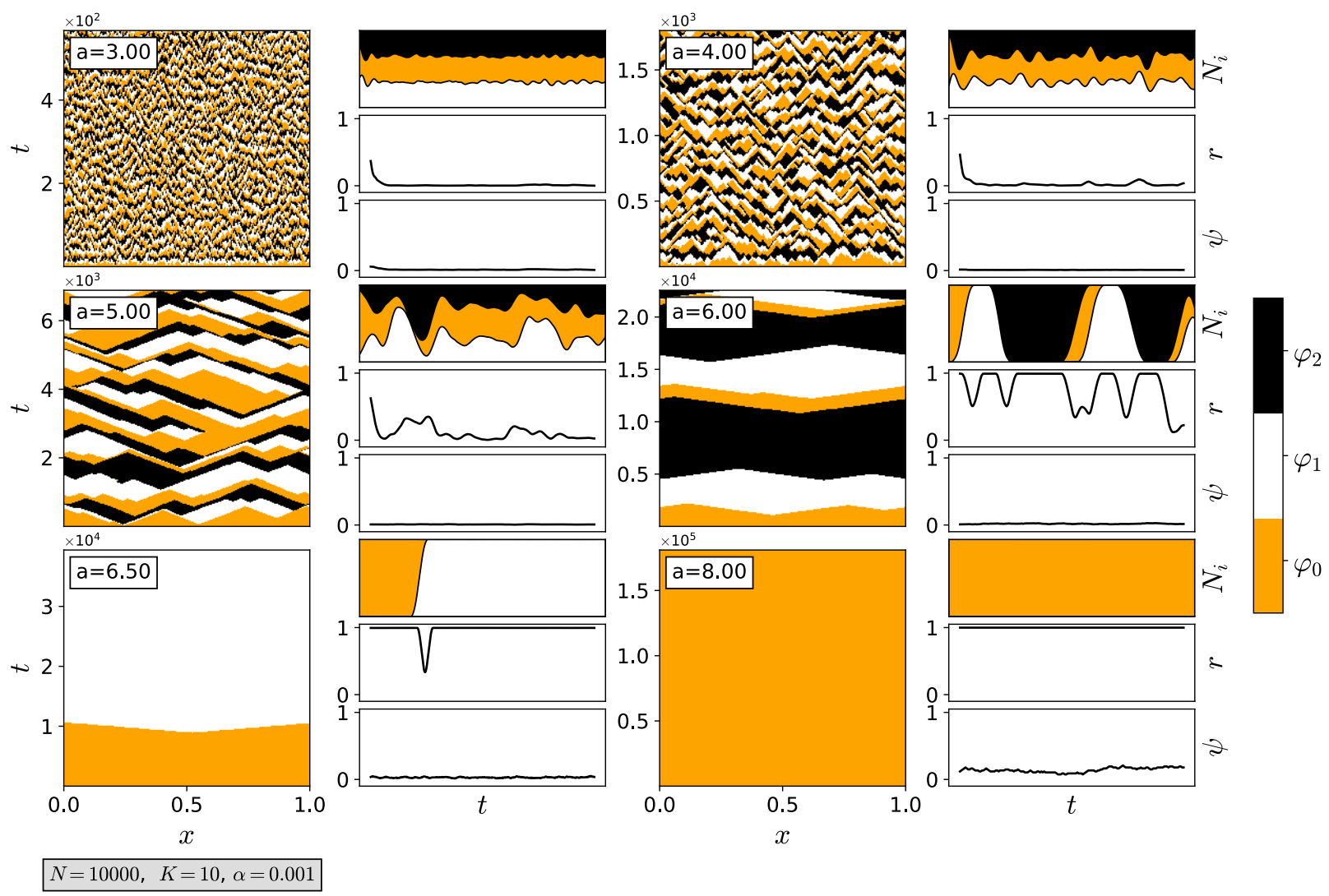

FIG. 9. Space-time plots, populations $N_{i}, \psi$ and $r$ order parameters. In all panels $N=10^{4}$, $K=10$ and $\alpha=10^{-3}$. The images on the left and center-right columns show space-time plots with position on the horizontal axis and time increasing upward. Adjacent and to the right of each space-time plot, three graphs show the corresponding populations, $\psi$, and $r$ as functions of time. Here we see that for a large system with low connectivity there are no regular oscillations. Instead, there are wave fronts that propagate and interfere and whose periods and amplitudes grow with $a$.

Fig. 8, such plots are shown for selected values of $\alpha t^{1}$ and system sizes up to $N=10^{4}$. In panel b) we see evidence of the GO transition for the value $\alpha=0.0017$ in the form of an inversion in curvatures for lines of constant coupling strength, which happens at $a_{c} \approx 2$. The inset in this same panel shows that for large $\alpha$ there is a clear split near the complete graph value $a_{c}=1.5$. In the case of the IP transition we observe that there is no upward curvature at any point, but rather a sharp increase in density of the lines of constant $a$ for higher values of $\alpha$, as seen in the bottom inset of Fig. 8 where the lines $a=3.375$ and

\footnotetext{
${ }^{1}$ See full animations of Fig. 8. GO transition, IP transition
} 
$a=3.306$ have collapsed to the same point near the origin.

Inspecting individual realizations of the dynamics in the small- $\alpha$ regime (Fig. 9) we see that the system never shows global synchronization. It instead exhibits wave-like patterns that propagate in both directions, similar to what is observed for large negative coupling [16], but here for $a$ positive. The amplitude and period of the wave increase with $a$ and with system size (for fixed $\alpha$ ), which suggests there is an IP phase in the limit $N \rightarrow \infty$.

\section{Initial-configuration dependence}

Up to this point, all the results discussed were obtained using uniform initial configurations (ICs). In Fig. 10 shows $\chi_{r}$ and $\chi_{\psi}$ for random ICs. The striking difference, compared to the results for uniform ICs, is the presence of a middle peak between the two identified previously. The scaling behaviors (panels $\mathbf{b}$ and $\mathbf{d}$ ) suggest that the effect persists for large system sizes if $\alpha$ is held constant. High values of $\chi$ result from multiple realizations of the
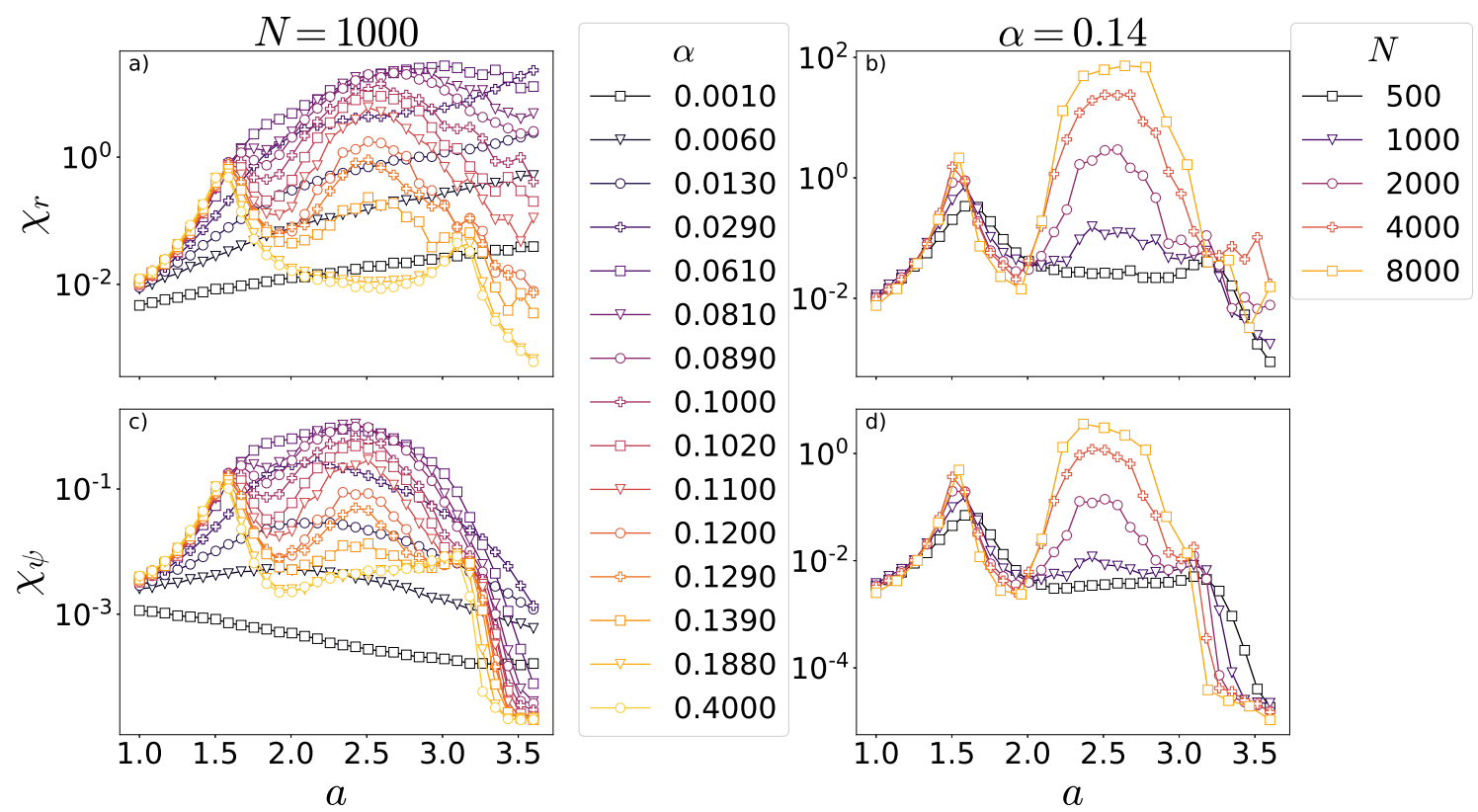

FIG. 10. (Color online) Scaled variances $\chi_{r}$ and $\chi_{\psi}$ for random initial conditions. Left column: fixed system size $N=1000$ with increasing $\alpha$. Right column: fixed $\alpha=0.14$ and increasing system sizes. Points represent an average over 4000 independent realizations with random initial configurations. 


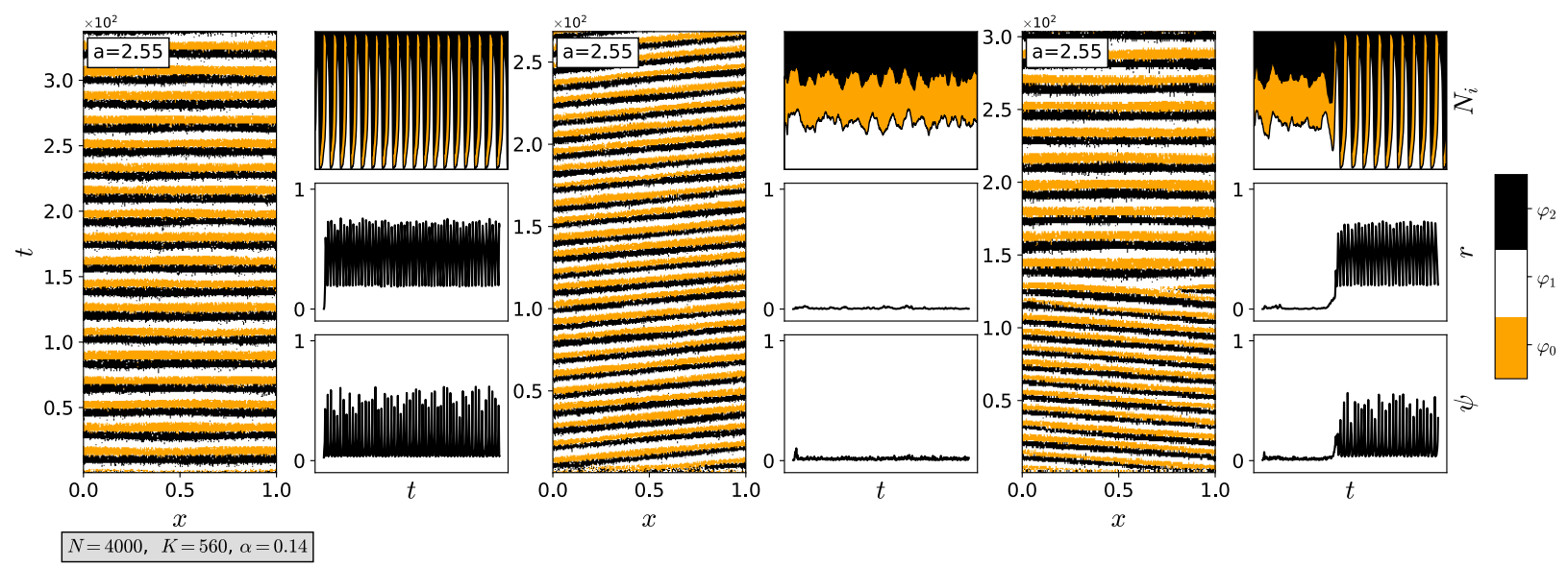

FIG. 11. Single realizations with random initial configurations. Left: Global oscillations. Middle: travelling wave. Right: fluctuation-induced change from a travelling wave to global oscillations. The fraction of realizations that converge to travelling waves is approximately $1.6 \%$. In all panels $N=4000, K=560, a=2.55$.

dynamics that produce net averages of the order parameter that differ from one to another. At the (continuous) GO and IP phase transitions, the values of the order parameter fluctuate strongly, giving rise to the peaks at $a=1.5$ and $a \approx 3.1$. Another situation which may lead to high $\chi$ values is a bistability between configurations that have large values of the order parameter and others having a small one, even when fluctuations associated with each configuration are small. This suggests a discontinuous phase transition where for some values of $\alpha$ the system can relax to multiple steady states.

Space-time plots of the dynamics with coupling $a \approx 2.5$ and $\alpha=0.14$ reveal that this is indeed the case (Fig. 11). Three examples are shown in Fig. 11, on the left is the familiar globally synchronized state, while the middle panel shows a travelling-wave state similar to those observed in [16], (but here, for large, positive coupling). The existence of a steady state with zero order parameter but $a>a_{c}$ is surprising since Fig. 10 suggests that wave-like solutions persist even in the limit $N \rightarrow \infty$ (with fixed $\alpha$ ), where interaction ranges become infinite.

The rightmost panel in Fig. 11 shows a fluctuation-induced change from a travelling wave to global synchrony. Such transitions allow the average order parameter to attain values between those associated with a wave state and a globally synchronized one, being 
closer to one or the other depending on what fraction of time it spent at that particular configuration. Starting from random ICs, about 1.6\% of realizations exhibit travelling waves, but since $\psi \approx 0$ for the waves and $\psi \sim \mathcal{O}(1)$ for the globally synchronized case, the variances $\chi_{r}$ and $\chi_{\psi}$ are sensitive even to small rates of occurrence. Due to fluctuations, waves do not persist in smaller systems; sizes $N \geq 700$ are required. Smaller systems exhibit either disordered phases with domains that increase in size and duration as the coupling grows, or global synchrony if the interaction range $K$ is large enough. In all cases, waves with exactly one spatial period over the system are observed, while for negative coupling, multiple stable wave numbers are found depending on the coupling magnitude [16].

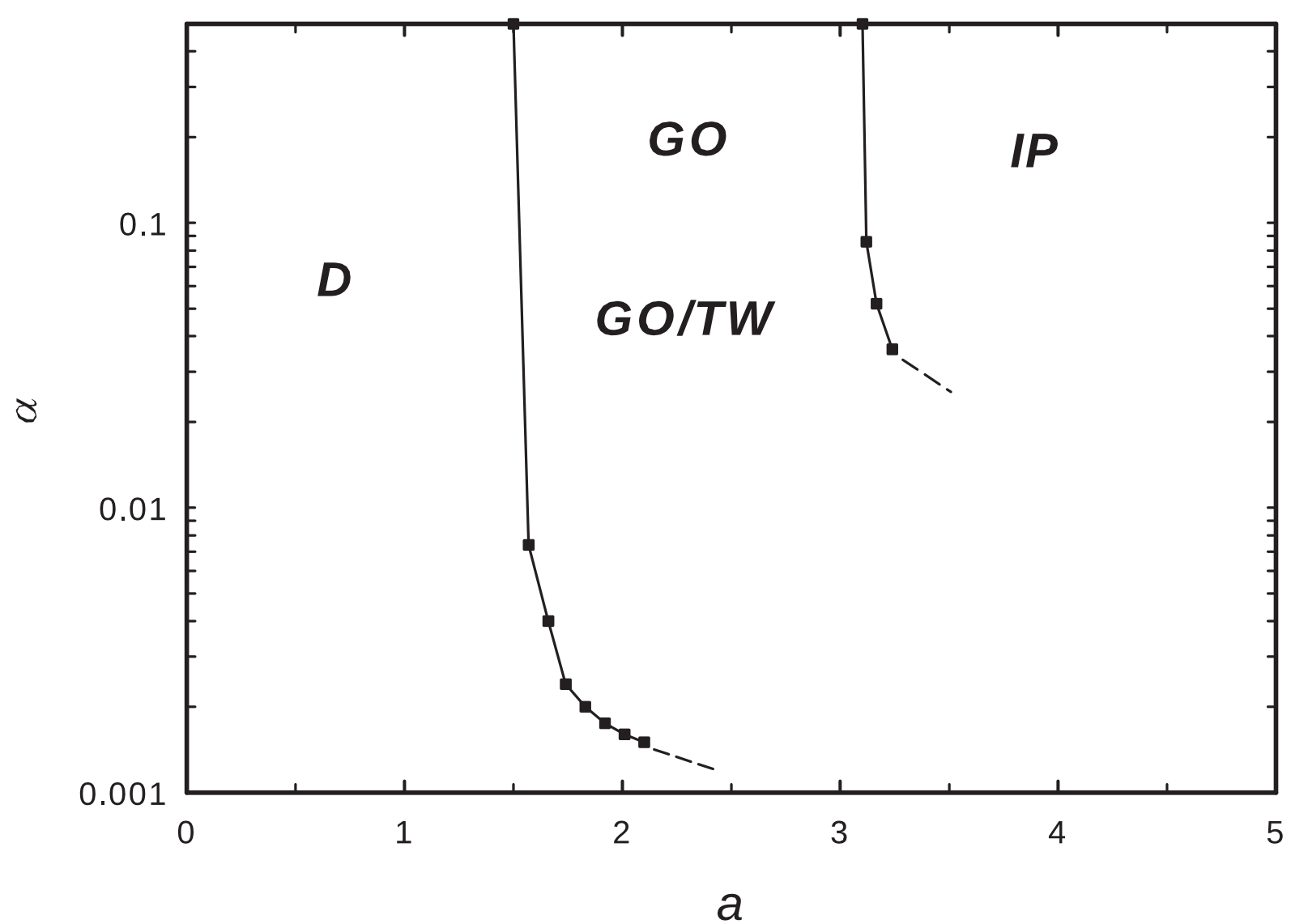

FIG. 12. Regular rings: Preliminary phase diagram in the $a-\alpha$ plane. Points are simulation results and (for $\alpha=0.5$ ) exact values. Lines are guides to the eye. Dashed lines represent our conjectures for how the phase boundaries continue. Phases: disordered (D); global oscillation (GO); travelling-wave (TW); infinite- period (IP). 
The existence of travelling waves can be understood by noting that, for $\alpha<0.5$, the system consists of $G=N / 2 K$ domains. $G$ represents the average path length for regular rings. (See Appendix VIA.) When $G$ is a multiple of three, the system is capable of containing a full wavelength without oscillators in the center of the domains. The wavefronts are then able to propagate as nucleation fronts [15] giving rise to travelling waves. In our simulations, with $N \leq 10^{4}$, we find only one stable wave-number (a wave with period $N$ ). This is in contrast with the wave patterns observed in [16], where multiple wave-numbers were identified as stable depending on the magnitude of the coupling strength.

Although travelling waves are rare starting from random ICs, initializing with solid blocks of $0 \mathrm{~s}, 1 \mathrm{~s}$ and $2 \mathrm{~s}$, each occupying one third of the system, the ensuing evolution consists of a stable travelling wave in nearly all cases. Thus the rarity for random ICs simply reflects the low probability of provoking a wave, and does not reflect an intrinsic instability of the travelling-wave state. ICs with smaller blocks, such that the system contains two or more waves, invariably yield, following a transient, a travelling wave whose wavelength equals the system size.

With the known phase transition points for $\alpha=0.5$ and the data from Figs. 10 and 8 (for $N \leq 10^{4}$ ) we are able to sketch the phase diagram (Fig. 12). While the phase boundaries are rather insensitive to changes in the connectivity for relatively large $\alpha$ values, they veer to larger couplings for small $\alpha$.

\section{THE WCM ON SMALL-WORLD NETWORKS}

Regular rings can be used as the starting point for constructing small-world networks, which are characterized by a small degree of separation between nodes while maintaining local regions tightly clustered. A well known algorithm for generating this type of network from regular rings was introduced by Watts and Strogatz [23]. Starting from a regular ring, for each edge in the graph, the clockwise node of that edge is swapped with probability $p$ for another randomly selected node, forbidding self-connections or repeated edges. Some care may be taken to avoid disconnected graphs as a result of this process, but this is unlikely for most parameter triplets $(N, K, p)$ considered here [23]. An example of such a rewired ring is shown in Fig. 13 .

To characterize a network as small-world, we define two quantities: 
- Mean path length $L$ : For nodes $i$ and $j$ in network $\mathcal{G}$, let $L_{i j}$ be the number of edges in the shortest path connecting these nodes. Then the average path length of $\mathcal{G}$ is $L=\left\langle L_{i j}\right\rangle$, where the average is over all pairs $(i, j)$ with $i<j$.

- Clustering coefficient $C$ : If node $i$ has $n_{i}$ neighbors, then the maximum possible number of connections among its neighbors is $m_{i}=\frac{n_{i}\left(n_{i}-1\right)}{2}$. Let $m_{i}^{*}$ be the number of connections among the neighbors of node $i$ in network $\mathcal{G}$. Then the clustering coefficient of $\mathcal{G}$ is $C=\left\langle\frac{m_{i}^{*}}{m_{i}}\right\rangle$, where the average is over all nodes $i$.

Evidently, the maximum possible value for $C$ is $C=1$ and the minimum possible value for $L$ is $L=1$. For networks generated by rewiring a regular ring graph, $C$ and $L$ are functions of the ring-graph parameters $N$ and $K$, as well as the rewiring probability $p$ : $L \equiv L(N, K, p), C \equiv C(N, K, p)$. As shown in the Appendix, for regular rings (i.e., $p=0$ ), we have:

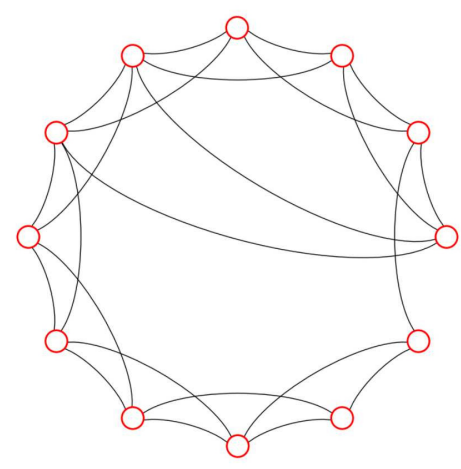

FIG. 13. Example of a rewired regular ring with $N=12, K=2$ and $p=0.1$.

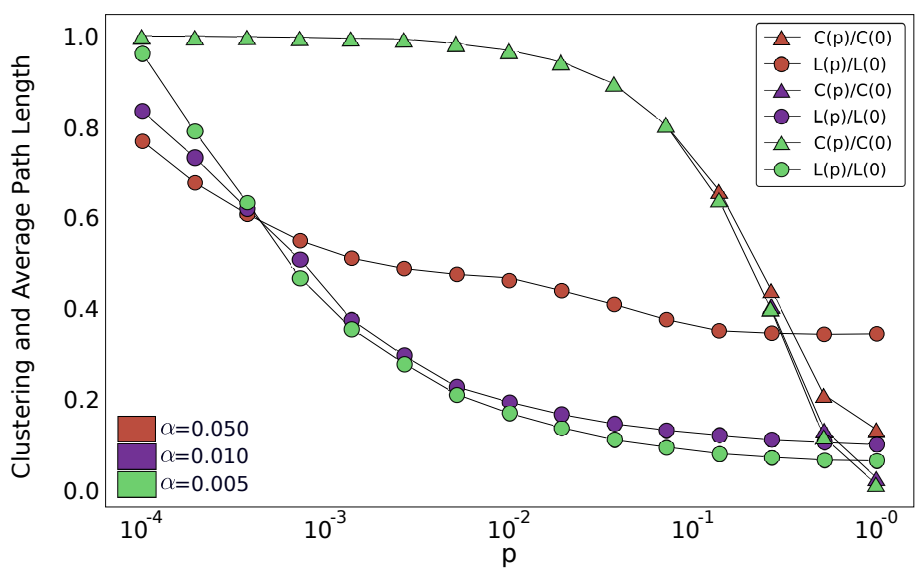

FIG. 14. (Color online) Clustering coefficient $C$ and mean path length $L$ versus rewiring probability $p$ for networks of $N=5000$ nodes. $K=250,50,25$ for red, blue, green curves respectively. The data represent averages over 400 independently generated networks. 

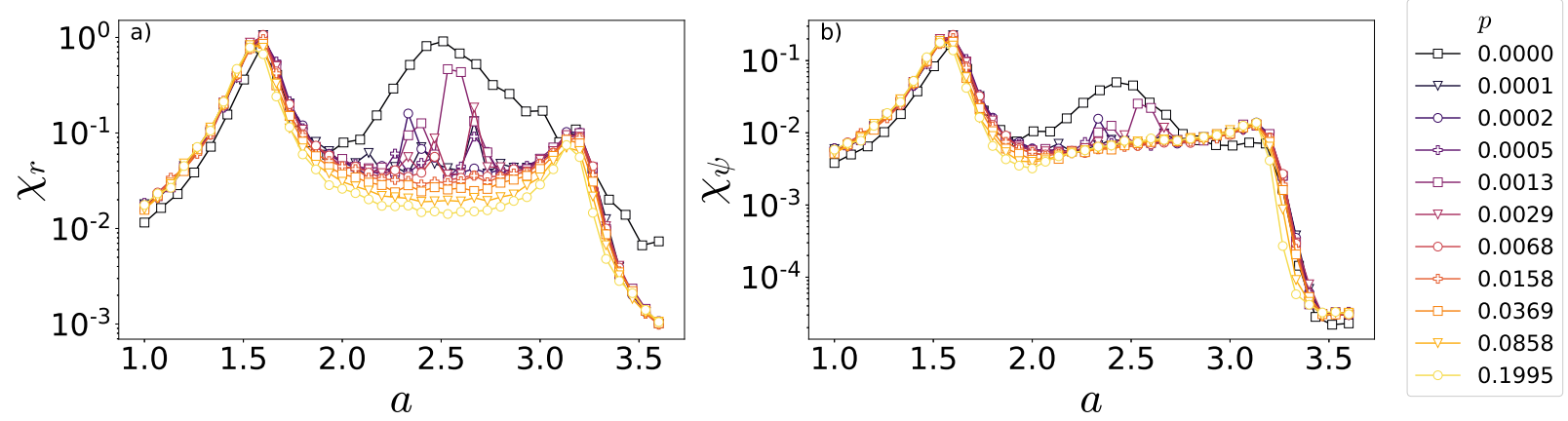

FIG. 15. (Color online) Small-world networks: Scaled variances, $\chi_{r}$ and $\chi_{\psi}$, for increasing rewiring probabilities. $N=1000, K=129, \alpha=0.129$ with random initial conditions. For $p \approx 0.01$ the curves follow their complete graph counterparts, even though the number of connections is half as many, and travelling waves become unstable.

$$
\begin{gathered}
C(N, K, 0)= \begin{cases}0, & \text { if } K<2 \\
\frac{3 K-3}{4 K-2}, & \text { if } 2 \leq K \leq \frac{N-1}{3} \\
\frac{12 K^{2}+6 K-6 K N+N^{2}-3 N+2}{4 K^{2}-2 K}, & \text { if } \frac{N-1}{3}<K<\frac{N}{2} \\
1, & \text { otherwise }\end{cases} \\
L(N, K, 0)= \begin{cases}\frac{K G(G-1)+r G}{N-1}, & \text { if } K \leq \frac{N}{2} \\
1, & \text { otherwise, }\end{cases}
\end{gathered}
$$

where $G$ is the largest integer smaller than $\frac{N-1}{2 K}$ or, using the floor operator,

$$
G=\left\lfloor\frac{N-1}{2 K}\right\rfloor .
$$

For nonzero values of $p$ we generate graphs and take the averages for $C$ and $L$, as shown in Fig. 14. For rewiring probabilities $p \in(0.001,0.1)$, rewiring preserves the clustering property while greatly reducing the average path length, thus characterizing small-world networks.

Since rewiring creates long-range interactions that reduce path lengths globally, we expect it to facilitate synchronization. This is shown to be the case in Fig. 15, where $p$ is gradually increased: realizations starting from random initial conditions are shown to readily synchronize for very small values of $p$, leading to the usual three phases identified for the complete graph. This means that the introduction of very few global connections is 


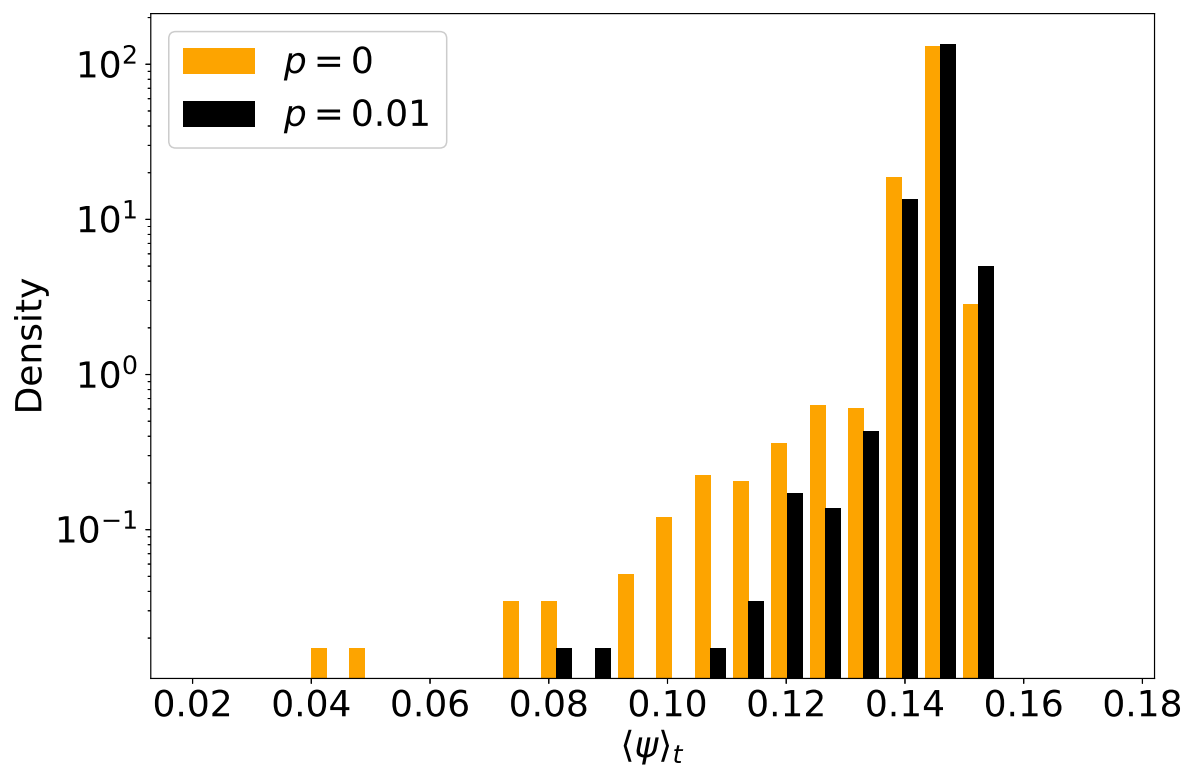

FIG. 16. (Color online) Networks with $N=1000, K=129$ and coupling $a=2.5$. Histograms of $\langle\psi\rangle_{t}$ for 9000 realizations without rewiring (yellow), and 9000 realizations with rewiring probability $p=0.01$ (black). The reduced frequency of small order-parameter values for $p=0.01$ compared with $p=0$ is evidence that rewiring destabilizes travelling waves, which are characterized by small values of $\psi$.

sufficient to destabilize travelling waves; they are virtually absent for $p \approx 0.01$. In Fig. 16 a histogram of the average order parameter per realization, $\langle\psi\rangle_{t}$, shows that the fraction of time spent in wave configurations is drastically reduced by the introduction of the rewiring procedure. When $p \geq 0.01$ and $a_{c}<a<a^{c}$, the system quickly converges to the GO phase even if it initially acquired a wave-like solution (see Fig. 17). Moreover, if system size is increased at constant $\alpha$, smaller values of $p$ are sufficient to cause the same effect, which is consistent with the fact that the number of long range connections is proportional to $N K$.

Following the same procedure as before, we plot in Fig. 18 the order parameters versus inverse system size $\lambda$. Here we fix $\alpha$ at a low value $(\alpha=0.0052)$ and vary $p$. 2 Comparing these results with panels d) and h) of Fig. 8 we see that the mean absolute value of $\psi$ is much greater when $p$ lies in the small-world region, indicating greater synchrony among oscillators. Space-time plots for values of $p>0$ show that indeed travelling waves become unstable, so

\footnotetext{
${ }^{2}$ See full animations of figure 18 GO transition, IP transition
} 


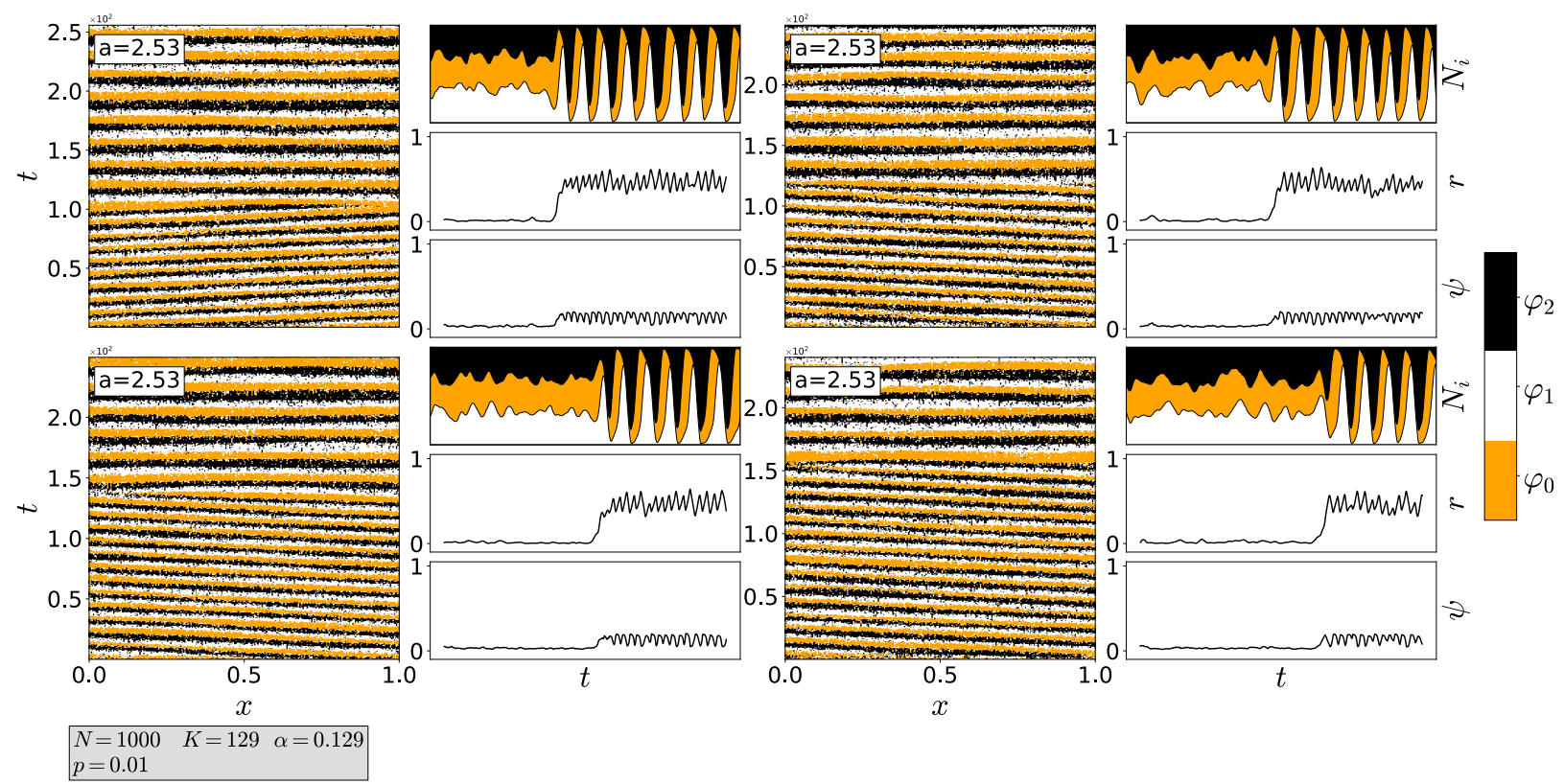

FIG. 17. Wave states for $N=1000, K=129, p=0.01$ and $a=2.53$. Only $0.04 \%$ of realizations (measured from 9000 realizations) with random initial configurations display traveling waves, which are also much more short-lived when compared to the $p=0$ equivalent system.

that the system exhibits only the three phases observed on the complete graph, namely, disordered, GO and IP phases. The phase diagram remains similar to the case of regular rings, displaying low sensibility to $\alpha$ except for very small values where the discreteness of finite systems becomes apparent. The main difference is that for any positive $p$ wave-like steady states are absent and thus the phase diagram contains only three macroscopically distinct phases. 

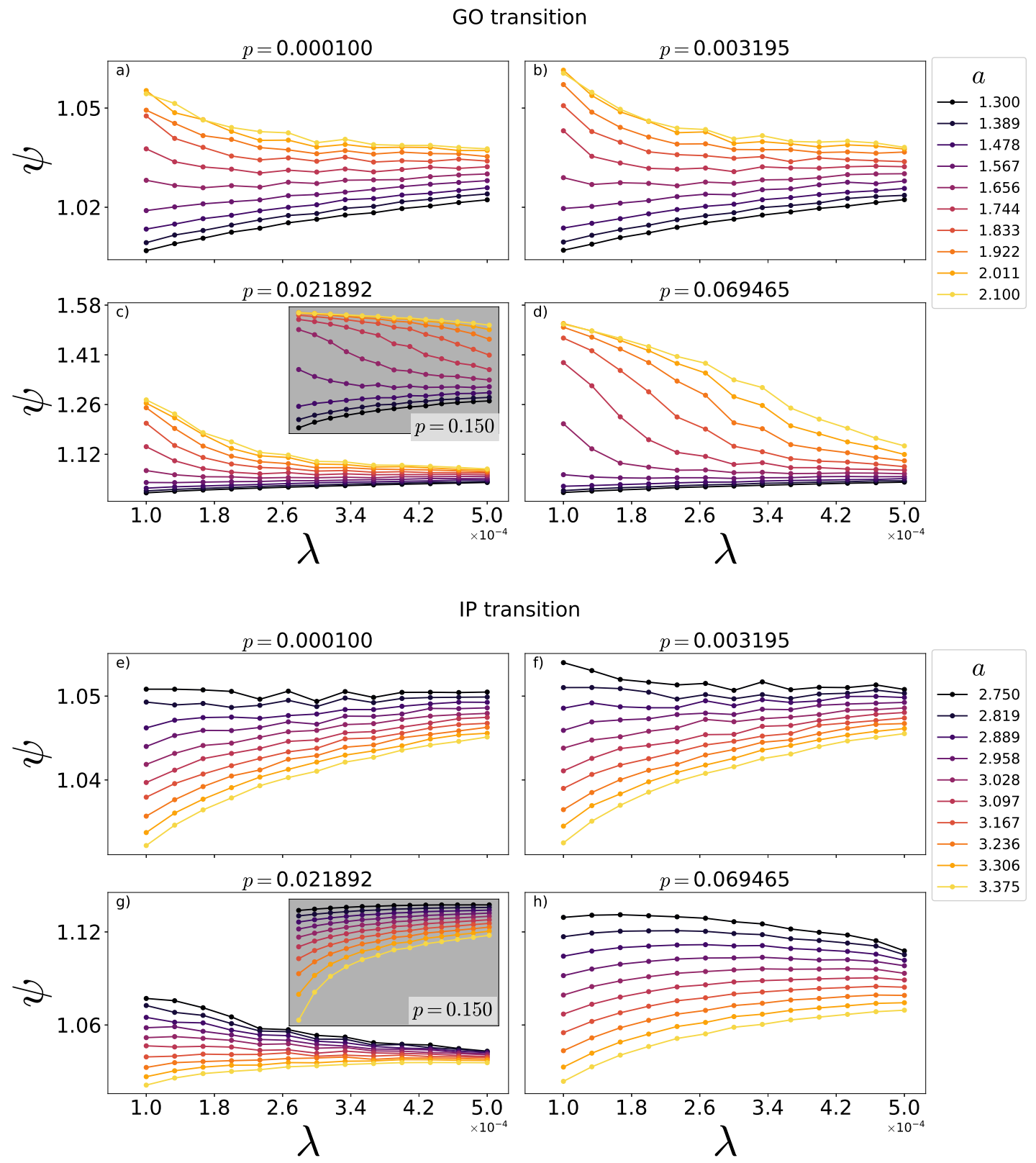

FIG. 18. (Color online) Order parameter $\psi$ versus inverse system size $\lambda$ for $\alpha=0.00524$ and various values of rewiring probability $p$ and couplings around the GO and IP transitions. Insets show the behavior for $p=0.15$, the highest rewiring probability used. 


\section{CONCLUSIONS}

We study the WCM model, a discrete-phase oscillator model exhibiting global synchronization and an infinite-period phase, on regular ring lattices and small-world networks. Each oscillator is coupled to a nonzero fraction $\alpha$ of the others, but the connectivity is in generally much smaller than for a complete graph. Our results support the conjecture that all three phases - disordered, globally synchronized, and infinite-period - appear for any nonzero connectivity $\alpha$.

Surprisingly, travelling waves also appear in the small- $\alpha$ regime on regular ring lattices; such waves may represent a long-lived metastable state. (A previous study identified waves in the anti-coupling case [16].) In our studies travelling waves constitute only about $1.6 \%$ of the steady states reached from starting from random initial configurations, and are prone to decay into global oscillations due to fluctuations when system size is small. The introduction of long-range interactions through rewiring (i.e., using the Watts-Strogatz algorithm) can lead to synchronization without increasing the total number of connections. Rewiring also suppresses travelling waves by introducing long range interactions.

The fact that regular rings are capable of sustaining travelling waves for $a>a_{c}$ is surprising, showing that networks of oscillators might fail to synchronize even in the presence of nonlocal interactions and strong coupling, conditions which are sufficient for the synchronization on hypercubic lattices of dimensions greater than 2 as well as on the complete graph. This raises the possibility that WMCs admit wave-like steady states on cubic lattices, similar to oscillations observed in the two-dimensional BelousovZhabotinsky reaction.

Several other questions remain open for future study, for example, whether travelling waves represent a stable phase for some range of parameters, or are always metastable, and whether waves of wavelength smaller than the system size $N$ are possible. We have sketched a phase diagram for ring lattices, but detailed information for the small-connectivity regime is lacking. Since the model exhibits a pair of continuous phase transitions, it is of interest to develop a full scaling picture, including the effect of ordering fields conjugate to the order parameters. Finally the question of what simple external perturbations are capable of destroying synchronization or the symmetry-broken phase may be of some practical interest. 


\section{APPENDIX}

\section{A. Path Length and Clustering of Regular Ring Lattices}

\section{Average Path Length}

The distance $L_{i j}$ between two nodes $i$ and $j$ is the minimum number of edges that must be traversed to connect them. The average path length is defined as the average distance between every possible pair of nodes in the network. For a network with $N$ nodes this is:

$$
L=\frac{2}{N^{2}-N} \sum_{i<j} L_{i j}
$$

Consider the lowest node in a ring graph with $N$ nodes and $2 K$ neighbors per node. Going counterclockwise $(\mathrm{CCW})$, there are $K$ nodes at distance 1, then $K$ nodes at distance 2 and so on until we reach some region near the top. In total, there will be $G$ groups of nodes, each with $K$ nodes, at distances $1,2,3 \ldots, G$ from our starting point at the bottom. $G$ is given by the largest integer smaller than $(N-1) /(2 K)$. This can be written with the floor operation:

$$
G=\left\lfloor\frac{N-1}{2 K}\right\rfloor
$$

This same procedure can be performed from the clockwise (CW) direction. Thus, there are $2 K$ nodes at distance 1 and so on up to distance $G$. The last group of nodes at the top is therefore at a distance $G+1$, but it contains less than $2 K$ nodes. Indeed, it contains a number $R$ of nodes equal to the remainder of the integer division of $N-1$ by $2 K$ :

$$
R=N-1-2 K G
$$

This reasoning can be visualized in Fig. 19 .

Since there are $N-1$ pairs between the bottom node and all other nodes in the lattice, the average distance $L_{0}$ between the bottom node and all other nodes is then given by: 


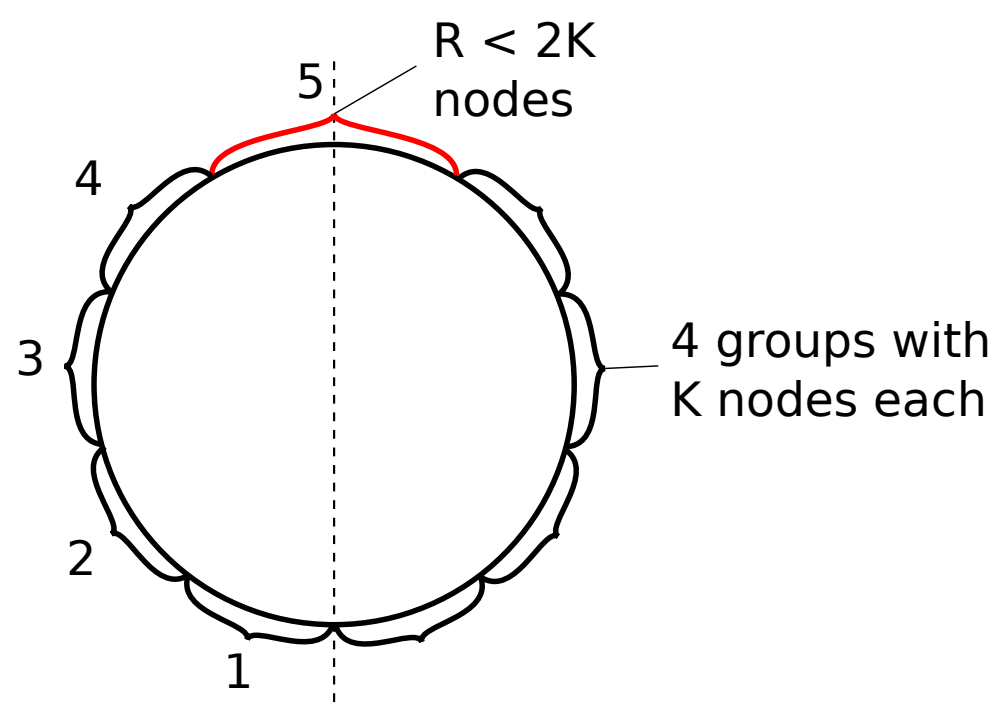

FIG. 19. Counting the number of nodes at distances visually. Here $G=4$ groups at distances 1, 2, 3,4 from the bottom node.

$$
\begin{aligned}
L_{0} & =\frac{1}{N-1}\left[2 K \sum_{i=1}^{G} i+R(G+1)\right] \\
& =\frac{1}{N-1}[K G(G+1)+R(G+1)] \\
L_{0} & =(G+1)\left(1-\frac{K G}{N-1}\right)
\end{aligned}
$$

where we used equation 12 to substitute in for $R$. Because we started with an arbitrary node at the bottom, this result is true for any given node in a regular ring, and thus we conclude that the average path length for the whole network is just $L_{0}$.

$$
\begin{array}{r}
L(N, K)=(G+1)\left(1-\frac{K G}{N-1}\right) \\
\text { with } \quad G=\left\lfloor\frac{N-1}{2 K}\right\rfloor
\end{array}
$$

\section{Average Clustering}

The clustering coefficient for a node $i$ in the graph is defined as: let $n_{i}$ be the number of neighbors of some node $i$. Then, there are at most $\left(n_{i}^{2}-n_{i}\right) / 2$ connections between any two 


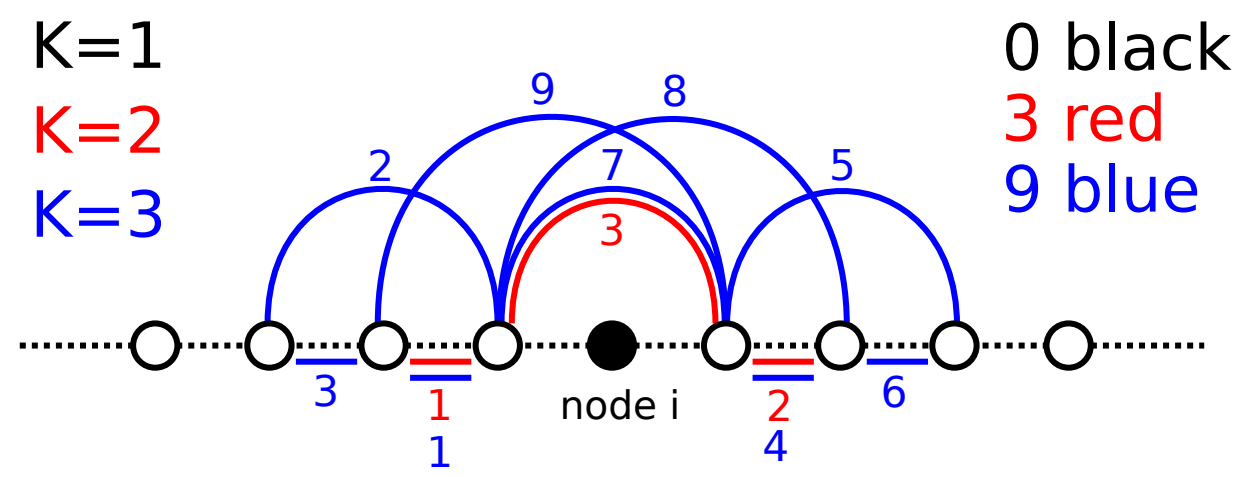

FIG. 20. (Color online) Counting $m$ for $k=1,2,3$. We note three contributions to $m$ : a fully connected group of $K \mathrm{CW}$ neighbors (left), a fully connected group of $K \mathrm{CCW}$ neighbors (right) and connections that go "over" the center node.

of its neighbors. Let $m_{i}$ be the number of actual connections that are present in a particular graph. Then, the clustering coefficient $C_{i}$ of node $i$ is given by:

$$
C_{i}=\frac{2 m_{i}}{n_{i}^{2}-n_{i}}
$$

If there are $N$ nodes in the graph, the average clustering coefficient is thus given by:

$$
C=\frac{1}{N} \sum_{i=1}^{N} C_{i}
$$

First, consider a "close-up" of a section of a regular ring where $N \gg K$. Consider a node $i$ with $K \mathrm{CW}$ neighbors and $K \mathrm{CCW}$ neighbors. Let $m$ be the number of connections between neighbors of node $i$. We can manually count $m$ for some values of $K$ :

$$
\begin{array}{lll}
m=0 & \text { if } & K \leq 1 \\
m=3 & \text { if } & K=2 \\
m=9 & \text { if } & K=3
\end{array}
$$

The general case for $N \gg K$ can be counted by summing the contributions of the three groups as shown in figure 20. Two fully connected groups of $K$ nodes each contributes with $\left(K^{2}-K\right)$ connections. The connections that bypass node $i$ also contribute with $\left(K^{2}-K\right) / 2$ connections and thus we have. 

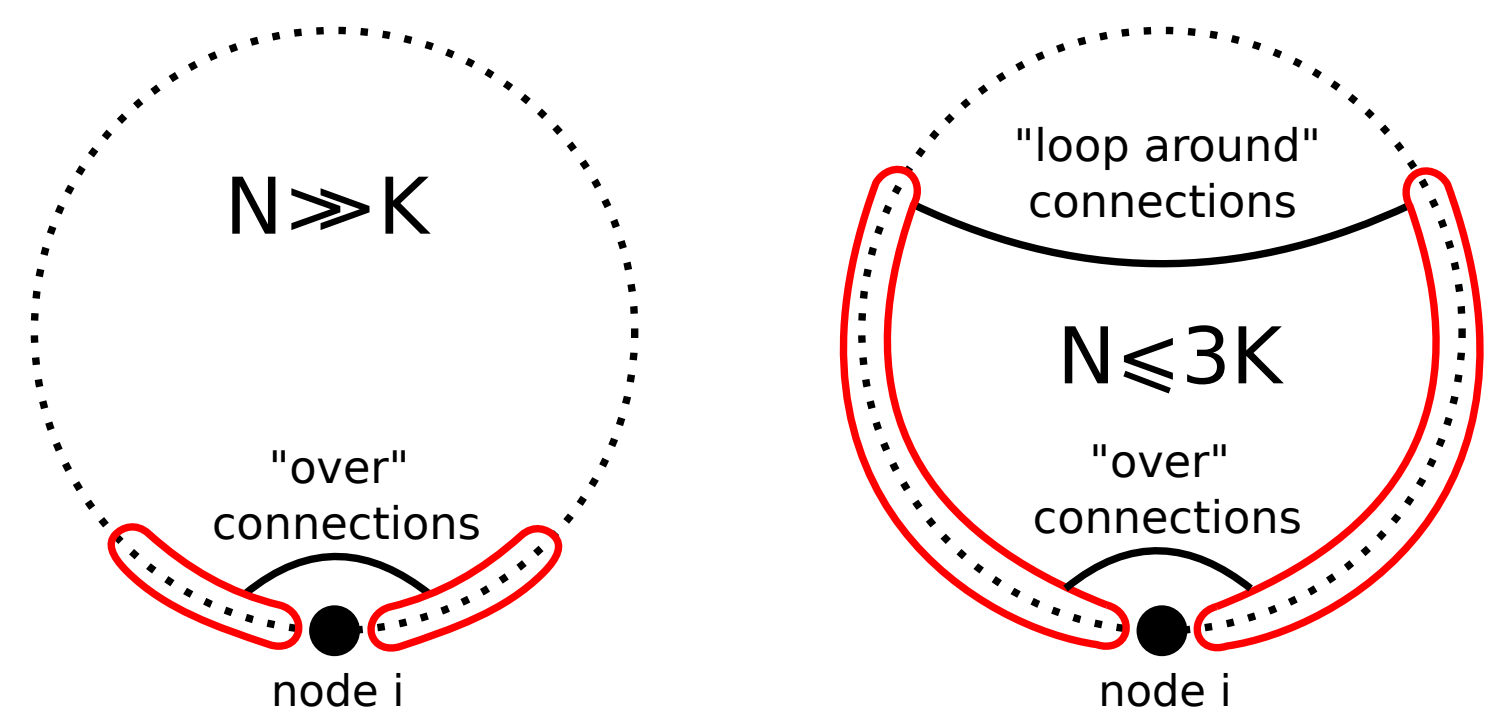

FIG. 21. Connections that contribute to the clustering coefficient of node $i$. The red regions represent the CW and CCW groups of neighbors of node $i$, and they are fully connected each within itself. Additional connections are made going "over" node $i$, but when $N \leq 3 K$ there are additional connections that loop around the opposite side of the lattice.

$$
m=\frac{3}{2}\left(K^{2}-K\right)
$$

Now we divide equation 17 by the total number of connections between the $2 K$ neighbors of node $i$ to get its clustering coefficient $C_{i}$ :

$$
C_{i}(N, K)=\frac{3 K-3}{4 K-2}
$$

When $N$ is not so large compared to $K$, additional connections between the CCW and CW neighbors may appear by looping around the opposite side of node $i$, as depicted in figure 21 . These additional connections will be present whenever the remaining nodes that are neither in the CW or CCW group are fewer than $K$. The number of such nodes is just $N-2 K-1$, which gives us the condition $N \leq 3 K$ for additional connections to be present.

The number of "loop around" connections that will be present will depend on how many nodes there are in the remaining group after removing node $i$ and its immediate neighbors. Let this number be denoted by $D=N-2 K-1$. Then, the number of additional connections will be given by 


$$
(K-D)+(K-D-1)+\ldots+2+1=\frac{(K-D+1)(K-D)}{2}
$$

Adding equation 19 to 17 we get

$$
m=\frac{3}{2}\left(K^{2}-K\right)+\frac{1}{2}(3 K-N+1)(3 K-N+2)
$$

And thus the clustering now becomes:

$$
C_{i}(N, K)=\frac{3 K-3}{4 K-2}+\frac{(3 K-N+1)(3 K-N+2)}{4 K^{2}-2 K}
$$

This formula holds up to the point when $D=0$ or $N=2 K+1$. For all values $D \leq 0$ the regular ring is in fact a complete graph, where every node connects to every other. In these cases the clustering coefficient is always equal to one. Formulas 18 and 21 together offer a complete expression for the clustering of node $i$. Since $C_{i}=C_{j} \forall i, j$, this is just the average clustering of the whole network and we finally get:

$$
C(N, K)= \begin{cases}\frac{3 K-3}{4 K-2} & \text { if } N>3 K \\ \frac{3 K-3}{4 K-2}+\frac{(3 K-N+1)(3 K-N+2)}{4 K^{2}-2 K} & \text { if } 3 K \geq N>2 K+1 \\ 1 & \text { else }\end{cases}
$$

\section{ACKNOWLEDGMENTS}

KLR acknowledges the financial support from CNPq and CAPES, Brazil. RD acknowledges support of CNPq under project 303766/2016-6. 
[1] Y. Kuramoto. Chemical oscillations, waves, and turbulence. Courier Corporation, 2003.

[2] S. H. Strogatz and I. Stewart. Coupled oscillators and biological synchronization. Scientific American, 269(6):102-109, 1993.

[3] S. H. Strogatz. From kuramoto to crawford: exploring the onset of synchronization in populations of coupled oscillators. Physica D: Nonlinear Phenomena, 143(1-4):1-20, 2000.

[4] S. H. Strogatz. Sync: How order emerges from chaos in the universe, nature, and daily life. Hachette UK, 2012.

[5] A. Pikovsky, M. Rosenblum, and J. Kurths. Synchronization: a universal concept in nonlinear sciences, volume 12. Cambridge university press, 2003.

[6] K. I. Tainaka. Lattice model for the lotka-volterra system. Journal of the Physical Society of Japan, 57(8):2588-2590, 1988.

[7] K. I. Tainaka. Stationary pattern of vortices or strings in biological systems: lattice version of the lotka-volterra model. Physical Review Letters, 63(24):2688, 1989.

[8] K. Tainaka and Y. Itoh. Topological phase transition in biological ecosystems. EPL (Europhysics Letters), 15(4):399, 1991.

[9] Y. Itoh and K. I. Tainaka. Stochastic limit cycle with power-law spectrum. Physics Letters A, 189(1-2):37-42, 1994.

[10] K. I. Tainaka. Vortices and strings in a model ecosystem. Physical Review E, 50(5):3401, 1994.

[11] K. Wood, C. Van den Broeck, R. Kawai, and K. Lindenberg. Universality of synchrony: Critical behavior in a discrete model of stochastic phase-coupled oscillators. Physical review letters, 96(14):145701, 2006.

[12] K. Wood, C. Van den Broeck, R. Kawai, and K. Lindenberg. Critical behavior and synchronization of discrete stochastic phase-coupled oscillators. Physical Review E, 74(3):031113, 2006.

[13] K. Wood, C. Van den Broeck, R. Kawai, and K. Lindenberg. Effects of disorder on synchronization of discrete phase-coupled oscillators. Physical Review E, 75(4):041107, 2007.

[14] K. Wood, C. Van den Broeck, R. Kawai, and K. Lindenberg. Continuous and discontinuous phase transitions and partial synchronization in stochastic three-state oscillators. Physical 
Review E, 76(4):041132, 2007.

[15] V. R. Assis, M. Copelli, and R. Dickman. An infinite-period phase transition versus nucleation in a stochastic model of collective oscillations. Journal of Statistical Mechanics: Theory and Experiment, 2011(09):P09023, 2011.

[16] D. Escaff, K. Lindenberg, et al. Arrays of stochastic oscillators: Nonlocal coupling, clustering, and wave formation. Physical Review E, 90(5):052111, 2014.

[17] Y. Kuramoto, T. Aoyagi, I. Nishikawa, T. Chawanya, and K. Okuda. Neural network model carrying phase information with application to collective dynamics. Progress of Theoretical Physics, 87(5):1119-1126, 1992.

[18] H. Ohta and S.-i. Sasa. Critical phenomena in globally coupled excitable elements. Physical Review E, 78(6):065101, 2008.

[19] S. Shinomoto and Y. Kuramoto. Phase transitions in active rotator systems. Progress of Theoretical Physics, 75(5):1105-1110, 1986.

[20] F. Rozenblit and M. Copelli. Collective oscillations of excitable elements: order parameters, bistability and the role of stochasticity. Journal of Statistical Mechanics: Theory and Experiment, 2011(01):P01012, 2011.

[21] K. Huang. Statistical mechanics, 2nd. Edition (New York: John Wiley \& Sons), 1987.

[22] M. Plischke and B. Bergersen. Equilibrium Statistical Physics. World Scientific, 1994.

[23] D. J. Watts and S. H. Strogatz. Collective dynamics of small-worldnetworks. Nature, 393 (6684):440, 1998. 